\title{
Transporting rats to the test situation on a cart can modify rat exploratory behavior in the elevated plus-maze
}

\author{
SILVIO MORATO and MARCUS L. BRANDÃO \\ Faculdade de Filosofia, Ciências e Letras de Ribeirão Preto-USP, Ribeirão Preto-SP, Brazil
}

\begin{abstract}
Male Wistar rats were submitted to a 24-h period of social isolation combined with a 24-h period of exposure to a different vivarium, of transportation to the test room either by cart or gently carried by hand, and immediate testing in an arena and in the elevated plus-maze. Results showed that transportation by cart was aversive inasmuch as it decreased exploratory behavior in the maze. They also suggested that exploratory behavior in the closed arms of the plus-maze was also modified by aversion. This trend was reversed when the rats were also socially isolated before testing. The data suggest that less intense anxiety, as caused by social isolation or cart transportation alone, trigger mechanisms that mediate less energetic behavior, while higher levels of anxiety (as produced by the two combined) trigger mechanisms that mediate more vigorous activity. These results are comparable to others from studies showing that rats switch from active to static to explosive behaviors with increasing intensities of aversion.
\end{abstract}

The elevated plus-maze test (Handley \& Mithani, 1984), a modified procedure originated by Montgomery (1955), has become a known model test of animal anxiety. It was reported by Pellow and coworkers (Pellow, Chopin, File, \& Briley, 1985) as a valid and reliable tool for measuring anxiety on the basis of extensive investigation analyzing several of its behavioral, physiological, and pharmacological aspects. It consists of exposing animals to a plus-shaped maze elevated above the floor, with two facing closed arms (i.e., walled and closed at the ends) and two facing open arms (i.e., not walled and not closed at the ends). A rat will explore both the closed and open arms, but will typically more frequently enter and stay longer in the closed arms. The preference ratio of open-to-closed arms, both for entries and duration of stays (Handley \& Mithani, 1984; Pellow et al., 1985), is taken as an index of anxiety - the higher the ratio, the lower the anxiety level.

This test has been used to investigate anxiolytic and anxiogenic compounds and to study the involvement of neurotransmitters in anxiety (e.g., Critchley \& Handley, 1987; Handley \& Mithani, 1984; Pellow \& File, 1986). In spite of the apparent simplicity of this test situation, the aversion to the open arms seems to be influenced by many factors, among them sex (Johnston \& File, 1991), preexposure to the maze (File \& Zangrossi, 1993; Griebel, Moreau, Jenk, Martin, \& Misslin, 1993; Treit, Menard, $\&$ Royan, 1993), raised edges in the open arms (Treit et al.,

This work was supported by Research Grant 400702/93-2 from Conselho Nacional de Desenvolvimento Científico e TecnológicoCNPq. Correspondence should be directed to S. Morato, Faculdade de Filosofia, Ciências e Letras de Ribeirão Preto-USP, Av. Bandeirantes, 3900, Ribeirão Preto-SP, 14040-901, Brazil (e-mail: silmorat@) usp.br).
1993), type of floor (Morato \& Castrechini, 1989), time of day at which testing occurs (Griebel et al., 1993), and environmental levels of illumination (Griebel et al., 1993; Morato \& Castrechini, 1989).

In previous reports, we have shown that treatments producing aversion add to the aversiveness of the open arms (Maisonnette, Morato, \& Brandão, 1993; Motta, Maisonnette, Morato, Castrechini, \& Brandão, 1992). In those experiments, social isolation for short $(<24 \mathrm{~h})$ or long ( 2 weeks) periods of time caused rats to explore the open arms less than did controls, an effect that could be reversed or attenuated by drugs with an anxiolytic effect. Similar results had already been reported with rats isolated for 6 weeks, and the findings have been associated with increased fearfulness manifested as decreased activity in the plus-maze (Jankowska, Pucilowski, \& Kostowski, 1991). More recently, we have suggested that novelty (in the form of sequential 24-h exposures to one, two, or three different vivaria) was an aversive procedure and that the combination of its effects with the ones provoked by social isolation for $24 \mathrm{~h}$ produced different types of behavior in the elevated plus-maze (Morato \& Brandão, 1995). Thus, low levels of aversion, such as those produced by social isolation alone, produced less energetic behavior, resulting in a decrease in exploratory behavior, while increased levels (social isolation plus novelty) restored exploratory behavior to control levels.

The present experiment was designed to investigate what effects the combination of social isolation and the means by which the animals were transported to the test situation would have on the exploratory activity of rats in the plus-maze. We decided to investigate the means of transportation because we had noticed that animals kept in a different vivarium and transported to the test room in a cart behaved differently from rats that were kept in the 
vivarium adjacent to the test room and had their cages carried by hand. The present experiment made the assumption that the trip in the cart could be aversive because of trepidation, noise, or whatever other characteristics might be associated with it.

\section{METHOD}

\section{Subjects}

Ninety-six male Wistar rats, weighing 180-200 g, were, unless stated otherwise, housed 6 to a plastic box $(40 \times 30 \times 17 \mathrm{~cm})$ with ad-lib food and water under a 12:12-h light:dark photoperiod (lights on at $0700 \mathrm{~h}$ ) for at least 3 days prior to the test. Twentyfour hours before testing, the experimental rats were moved a distance of $45 \mathrm{~m}$ to a different vivarium that had a similar environment. Control rats remained in the vivarium adjacent to the test room. Room temperature was maintained at $24^{\circ}-26^{\circ} \mathrm{C}$ in both vivaria. All testing was performed between 0730 and $1030 \mathrm{~h}$.

\section{Apparatus}

Locomotor activity was measured for $5 \mathrm{~min}$ in a wooden arena $(60 \times 60 \times 30 \mathrm{~cm}$ with the floor divided into $15-\mathrm{cm}$ squares $)$; previous studies had shown this procedure to increase baseline exploratory activity in the plus-maze (Pellow et al., 1985). Then exploratory activity was observed in an elevated plus-maze similar to that described by Pellow et al.

Briefly, the maze, situated $50 \mathrm{~cm}$ from the floor, consisted of two open arms (with $1-\mathrm{cm}$ Plexiglas edges) and two closed arms (with $40-\mathrm{cm}$-high wooden walls and no roof) arranged such that like arms were opposite each other.

The arena and the maze were located in a room lit during the sessions by a $15-\mathrm{W}$ light bulb $1.75 \mathrm{~m}$ above the central part of the maze. All data were recorded using standard Grason-Stadler electromechanical equipment in the test room.

The cart was $90 \mathrm{~cm}$ long, $50 \mathrm{~cm}$ wide, and $85 \mathrm{~cm}$ high, with four $8-\mathrm{cm}$-diam hard rubber wheels. The cages were always transported in the top shelf $(85 \mathrm{~cm}$ from the floor $)$ of the cart, side by side and never on top of each other. The cart was always pushed by the same person.

\section{Procedure}

Housing. All rats were submitted to a minimum 3-day habituation period in a vivarium adjacent to the experimental room. After the habituation period, the experimental rats were transferred to a different vivarium (D) for $24 \mathrm{~h}$ while controls remained in the same place (S). During these $24 \mathrm{~h}$, half of both the D and $\mathrm{S}$ animals were put into individual plastic cages $(27 \times 15 \times 14 \mathrm{~cm})$ with ad-lib water and food (isolated rats, I), while the other half were kept in groups of 6 as described earlier (grouped rats, G). After this period, all rats were tested in the arena and immediately afterward in the elevated plus-maze. Thus, the subjects were divided into eight groups of 12 rats each.

Transportation. Half of the GD and ID rats were transported $45 \mathrm{~m}$ to another vivarium and $24 \mathrm{~h}$ later back to the testing room in a cart. On the way, there were four locations where the floor level differed by $2-3 \mathrm{~cm}$. The remaining half of the $\mathrm{D}$ animals were moved as gently as possible in their hand-carried cages. A similar procedure was repeated with $\mathrm{S}$ rats, except that they were transported by cart or carried gently by hand half the distance traveled by the $\mathrm{D}$ animals, and then were returned. Cart-transported $\mathrm{S}$ animals had to go over two floor-level differences in half the distance the D animals had to travel, and then over the same two floor-level differences on their way back. This ensured that both experimental and control animals traveled the very same distance and were exposed to similar stimuli. Apart from the differences in floor level, the floor was smooth and without cracks that could make the cart bump. When in movement, the metal vibrated and the cart made a low rumbling noise.

Behavioral measures. Each rat was individually placed in the arena for $5 \mathrm{~min}$ (where number of squares crossed were counted) and then immediately transferred to the elevated plus-maze, where it was gently placed with its nose facing one of the closed arms. The number of entries and time spent in each type of arm were recorded for $5 \mathrm{~min}$ by an experimenter, who stood still and away from the maze. An entry was recorded when all four paws were placed inside one arm. The number and durations of incomplete entries into the open arms were also recorded. This activity occurred mainly in the central square, with the animals typically coming from one of the closed arms, and involved such action as pendular movements of the head, head dipping down the sides of the open arms, and sniffing. The latency of the first entry was also recorded. This last measure and the count of squares crossed in the arena were used to assess locomotor deficits, if any. The recording equipment was programmed in such a way as to keep time records mutually exclusive, so that adding latency of first entry, duration of incomplete entries, and time spent in both types of arms would yield exactly $300 \mathrm{sec}$.

For analysis, the percentages of the number of entries made and the time spent in the open arms were calculated in relation to, re-
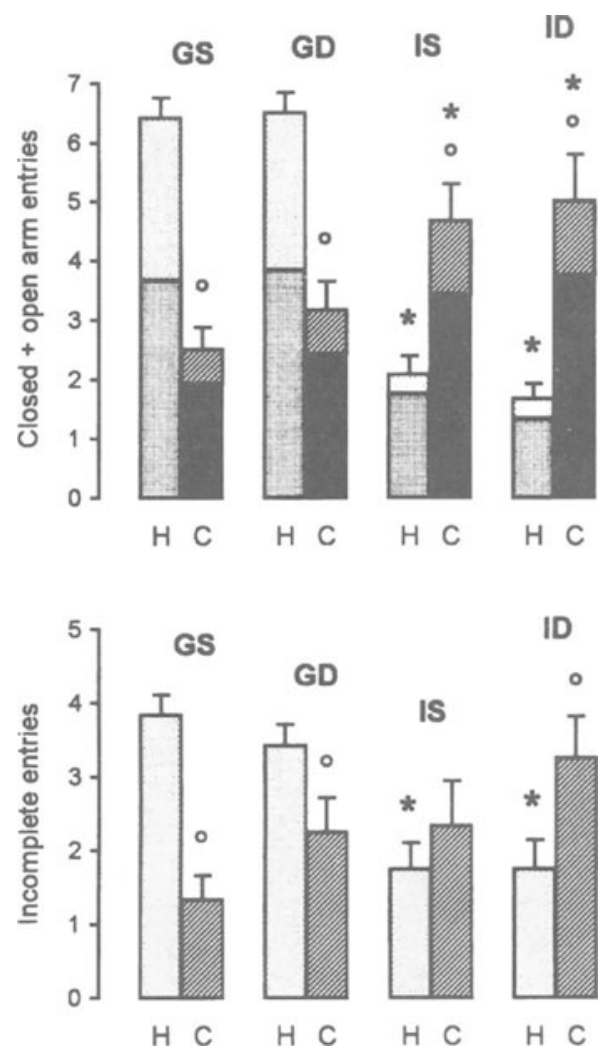

Figure 1. Effect of housing and grouping conditions on the exploratory activity of hand- or cart-transported rats. (Top) Number of entries into both open (upper, lighter part of the columns) and closed arms (lower, darker part of the columns). SEs were calculated from the total number of entries. (Bottom) Frequency of incomplete entries into the open arms. GS, grouped, same vivarium; GD, grouped, different vivarium; IS, isolated, same; ID, isolated, different; $H$, hand-transported; $C$, cart-transported. * Different from the corresponding grouped rats (Dunnett multiple comparisons test, $p<.01){ }^{\circ}$ Different from the corresponding hand-transported group (unpaired $t$ test, $p<.01$ ). 
spectively, total entries into both arms and total duration of the session.

\section{Statistical Analysis}

Comparisons between the different housing (same or different vivarium) and grouping (grouped or isolated) conditions were performed using analysis of variance and post hoc comparisons between groups, when necessary, done by the Dunnett multiple comparisons test, using GS animals as controls (Bruning \& Kintz, 1977). Whenever there was an increase or a decrease in both openand closed-arm entries, analysis of covariance was performed to determine whether the increase or reduction in the former was independent of any effect on the latter. Analysis was carried out with entries into the open arms as the dependent variable and entries into the closed arms as the covariant. A significant effect indicated that the alteration in open-arm entries was independent of effects on closed-arm entries. Within each condition, differences between hand-transported and cart-transported animals were assessed with unpaired $t$ tests.

\section{RESULTS}

\section{Total Activity}

General exploratory activity in the elevated plus-maze by rats submitted to the different housing and grouping conditions and either hand or cart transported is shown in Figure 1 and Table 1. It can be seen in the figure (top) that social isolation decreased the total number of entries in both arms by hand-transported subjects $[F(3,43)=72.099, p<.001]$. This overall decrease was due to significant decreases in both open-arm $[F(3,43)=69.710, p<.001]$ and closed-arm $[F(3,43)=$ $37.679, p<.0001]$ entries, which, however, were not concomitant, as analysis of covariance did not reveal a significant effect of housing $[F(1,43)=7.463, p<.01]$. Total entries by isolated cart-transported animals, by contrast, increased $[F(3,43)=4.062, p<.05]$ due to significant increases in closed-arm entries $[F(3,43)=$ $3.667, p<.05]$; open-arm entries did not differ significantly $[F(3,43)=2.101, p>.05]$. Transporting the rats in a cart instead of by hand decreased the total number of entries made by GS rats $[t(22)=7.727, p<.001]$ and GD rats $[t(22)=5.606, p<.001]$ at the same time that it in- creased this measure in IS rats $[t(22)=3.665, p<.01]$ and ID rats $[t(22)=4.022, p<.001]$.

The number of incomplete entries (Figure 1, bottom) was decreased by social isolation in hand-transported animals, both IS and ID $[F(3,44)=11.107, p<.001]$ in relation to the respective GS control animals. Cart-transported IS and ID rats, on the other hand, did not differ from the respective GS controls $[F(3,44)=2.420, p>.05]$. The $t$ test indicated that incomplete entry values for carttransported rats were significantly lower than those for hand-transported animals in Groups GS $[t(22)=5.823$, $p<.001]$ and GD $[t(22)=2.142, p<.05]$, not different for the IS groups $[t(22)=0.8318, p>.05]$, and higher for the ID animals $[t(22)=2.180, p<.05]$.

Table 1 shows that the number of crossings in the arena did not differ for hand-transported $[F(3,43)=$ $0.5244, p>.05]$ and cart-transported rats $[F(3,43)=$ $0.5296, p>.05]$. Neither was it different when we compared hand-and cart-transported rats in the GS $[t(22)=$ $0.96 .3, p>.05], \mathrm{GD}[t(22)=0.5745, p>.05]$, IS $[t(22)=$ $0.6009, p>.05]$, and ID groups $[t(22)=1.025, p>.05]$.

The table also shows that the latency of first entry did not differ for hand-transported animals $[F(3,43)=1.266$, $p>.05]$ but was slightly increased in ID cart-transported rats $[F(3,43)=4.328, p<.01]$. Comparison between the two means of transportation showed that there was no difference between the rats in Groups GS $[t(22)=1.025$, $p>.05], \mathrm{GD}[t(22)=0.4419, p>.05]$, and IS $[t(22)=$ $0.7153, p>.05$ ]; cart-transported animals, however, had higher latencies than did their hand-transported counterparts $[t(22)=3.279, p<.05]$.

\section{Arm Exploration}

Figure 2 shows the frequency of entries in both types of arms in terms of percentages. It can be seen that handtransported isolated animals, both $\mathrm{S}$ and $\mathrm{D}$, displayed a decrease (top) in the percentage of open-arm entries $[F(3,43)=21.361, p<.001]$ and an increase (bottom) in the percentage of closed-arm entries $[F(3,43)=21.041$, $p<.001]$. Cart-transported animals, on the other hand, did not differ in the percentages of either open- $[F(3,43)=$

Table 1

Frequency of Square Crossings in the Arena and Latency of the First Entry by Hand- or Cart-Transported Rats, According to Grouping and Housing Conditions

\begin{tabular}{|c|c|c|c|c|c|c|c|c|}
\hline \multirow[b]{3}{*}{ Transportation } & \multicolumn{4}{|c|}{ Grouped } & \multicolumn{4}{|c|}{ Isolated } \\
\hline & \multicolumn{2}{|c|}{ Same } & \multicolumn{2}{|c|}{ Different } & \multicolumn{2}{|c|}{ Same } & \multicolumn{2}{|c|}{ Different } \\
\hline & $M$ & $S E$ & $M$ & $S E$ & $M$ & $S E$ & $M$ & $S E$ \\
\hline \multicolumn{9}{|c|}{ Frequency of Crossings in the Arena } \\
\hline By hand & 62.25 & 1.80 & 62.17 & 1.64 & 64.25 & 1.76 & 63.42 & 1.99 \\
\hline By cart & 62.87 & 1.76 & 63.58 & 1.84 & 62.75 & 1.77 & 60.42 & 2.15 \\
\hline \multicolumn{9}{|c|}{ Latency of First Entry (in Seconds) } \\
\hline By hand & 5.75 & 0.45 & 5.75 & 0.35 & 5.50 & 0.31 & 4.92 & 0.30 \\
\hline By cart & 5.17 & 0.17 & 6.00 & 0.44 & 5.83 & 0.35 & 7.50 & $0.74^{*}+$ \\
\hline
\end{tabular}

Note- ${ }^{*}$ Different from the respective GS animals (Dunnett multiple comparisons test, $p<.01)$. Different from the hand-transported group isolated in the different vivarium (unpaired $t$ test, $p<.01$ ). 

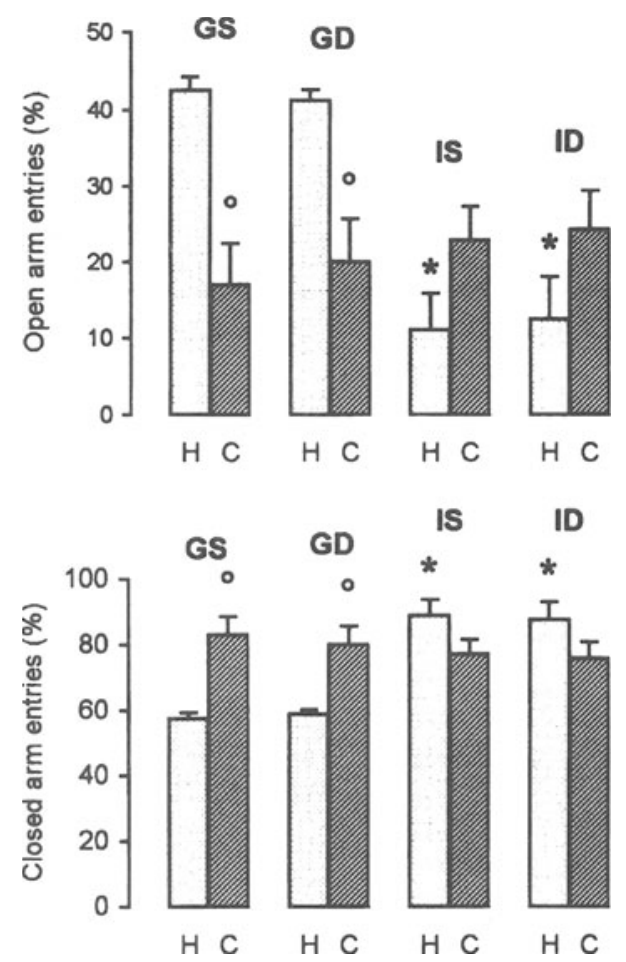

Figure 2. Percentage of entries into open (top) and closed (bottom) arms. GS, grouped, same vivarium; GD, grouped, different vivarium; IS, isolated, same; ID, isolated, different; $H$, handtransported; $C$, cart-transported. *Different from the animals grouped in the same vivarium (Dunnett multiple comparisons test, $p<.01)$. ${ }^{\circ}$ Different from the corresponding hand-transported groups (unpaired $t$ test, $p<.01$ ).

$0.4021, p>.05]$ or closed-arm entries $[F(3,43)=0.4021$, $p>.05]$. Grouped cart-transported animals exhibited a decrease in percentages of open-arm entries [GS, $t(22)=$ $4.497, p<.001 ; \mathrm{GD}, t(22)=3.649, p<.01]$ and an increase in the percentages of closed-arm entries [GS, $t(22)$ $=4.477, p<.001 ; \mathrm{GD}, t(22)=3.598, p<.01]$. Transportation did not affect either open-arm $[t(22)=1.852, p>$ $.05]$ or closed-arm entry percentages $[t(22)=1.852, p>$ $.05]$ by IS rats, nor did it affect open- $[t(22)=1.574$, $p>.05]$ or closed-arm entry percentages $[t(22)=1.574$, $p>.05]$ by ID rats.

Figure 3 (top and middle) shows the occupation of both types of arms. As can be seen, the hand-transported isolated animals spent less time in the open arms than did the grouped ones $[F(3,43)=31.773, p<.001]$, while cart-transported rats exhibited no differences $[F(3,43)=$ $1.243, p>.05]$. Transportation by cart decreased time in the open arms for both GS $[t(22)=3.542, p<.01]$ and $\operatorname{GD}[t(22)=7.158, p<.001]$ animals, while it increased time in the open arms for both IS $[t(22)=2.938, p<.001]$ and ID $[t(22)=2.341, p<.01]$ rats. Unlike with the open arms, analysis of exploratory activity in the closed arms showed that hand-transported isolated animals spent more time in the closed arms than did the grouped ones $[F(3,43)=32.059, p<.001]$, while cart-transported rats exhibited no differences $[F(3,43)=1.931, p>.05]$. Use of the cart increased the time spent in the closed arms by Groups GS $[t(22)=4.908, p<.001]$ and GD $[t(22)=$ $5.456, p<.001]$, did not affect Group IS $[t(22)=1.800$, $p>.05]$, and decreased the time spent there by Group ID $[t(22)=3.463, p<.01]$.

Figure 3 (bottom) shows the time spent by the rats in making incomplete entries into the open arms. Social isolation caused decreases in the time spent by the handtransported rats $[F(3,43)=11.386, p<.001]$ but not that by the cart-transported animals $[F(3,43)=2.026, p>$ $.05]$. Transportation by cart decreased the time spent on
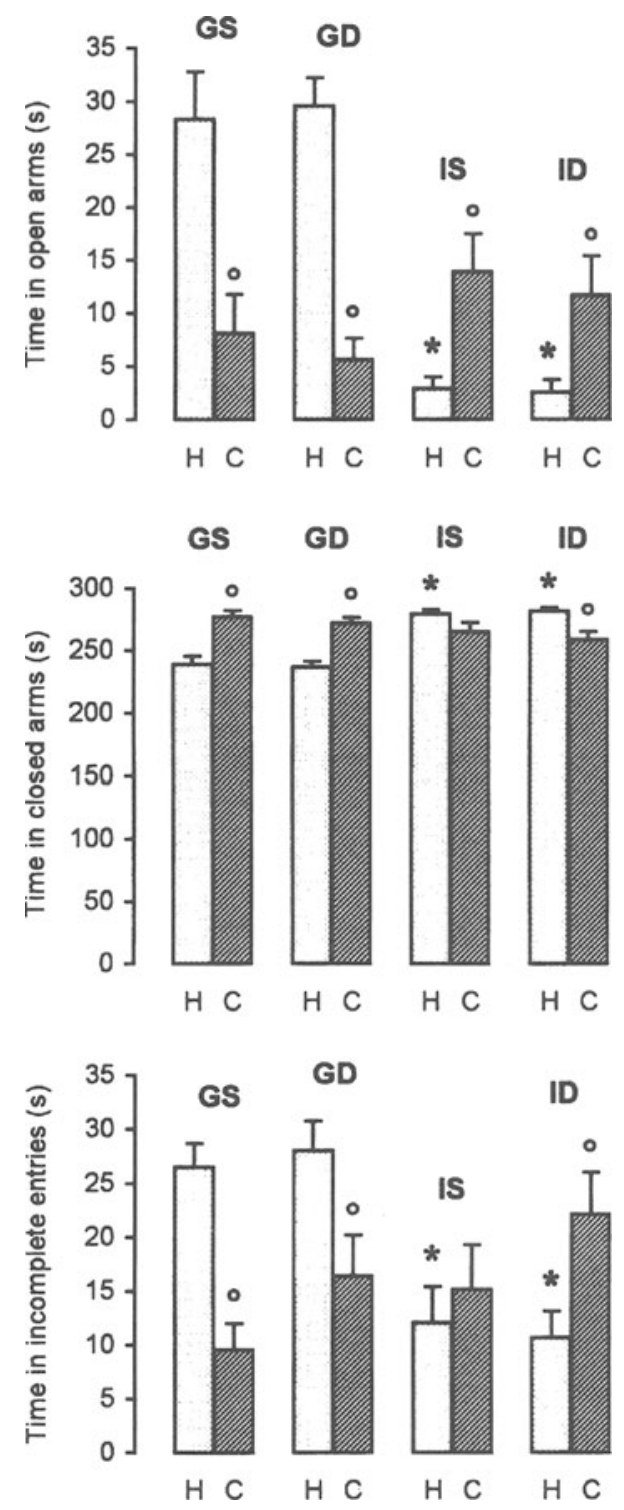

Figure 3. Time spent in the open (top) and closed (middle) arms and in incomplete entries into open arms (bottom). GS, grouped, same vivarium; GD, grouped, different vivarium; IS, isolated, same; ID, isolated, different. $H$, hand-transported; $C$, cart-transported. *Different from the corresponding GS group (Dunnett multiple comparisons test, $p<.01)$. ${ }^{\circ}$ Different from the corresponding hand-transported group (unpaired $t$ test, $p<.05$ ). 
incomplete entries by Groups GS $[t(22)=5.122, p<$ $.001]$ and $\mathrm{GD}[t(22)=2.490, p<.05]$, did not affect that of Group IS $[t(22)=0.5863, p>.05]$, but increased that of Group ID $[t(22)=2.463, p<.05]$.

\section{DISCUSSION}

The effects of social isolation on hand-transported animals were similar to those already described in the literature: socially isolated rats decreased their exploration of the open arms, in terms of both number of entries and time taken, and the frequency of entries into the closed arm (Jankowska et al., 1991; Maisonnette et al., 1993; Morato \& Brandão, 1995; Motta et al., 1992). These results were independent of the rats' being habituated in the vivarium adjacent to the test room (S) or in another vivarium farther away (D).

Transporting the animals by cart caused a decrease in the total number of entries (Figure 1), entries (Figure 2) and time (Figure 3 ) spent in the open arms by grouped animals (GS and GD), while paradoxically increasing these measures in socially isolated animals (IS and ID) in comparison with the respective hand-transported groups. These effects probably cannot be attributed to locomotor alterations, since the number of square crossings in the arena and the latency of first entry were hardly altered, in spite of the fact that this only indicates that there was no motor impairment. By itself, both isolation and cart transportation decreased total exploratory activity; the combination of both restored exploratory behavior, although not to the levels observed in the hand-transported grouped subjects.

The use of a cart for transporting laboratory rats is probably an aversive manipulation, as judged by the decrease in exploratory behavior observed with the grouped animals, which was similar to the decrease caused by social isolation alone, observed both here and elsewhere (Maisonnette et al., 1993; Morato \& Brandão, 1995; Motta et al., 1992). In these studies, decreases in exploratory activity (as judged by decreased locomotion) were observed in both open and closed arms. The rats that were submitted to the most severe conditions, either environmentally (longer isolation periods) or pharmacologically (anxiogenic drugs), usually made only one entry into one of the closed arms and remained there for the whole session. In the less severe conditions, however, only the exploration of the open arms decreased. In general, measures obtained in the closed arms are not connected to decreases due to anxiety, being taken into account only as a component of total entries. The only exception to this view is a report by Cruz, Frei, and Graeff (1994) stating that the time spent in the closed arms is a mirror image of that spent in the open arms and thus also indicates anxiety in the elevated plus-maze. We propose that when previous treatments are severe enough, the measures obtained in the closed arms, frequency and time, may indicate increased anxiety at a time when measures in the open arms are already reduced to zero.
It is not quite clear why the addition of the effects of social isolation and cart transportation (two manipulations clearly aversive in this model) increased exploratory behavior instead of reducing it even more. The same phenomenon has recently been reported (Morato \& Brandão, in press) for social isolation and novelty in the form of exposure to a novel vivarium each $24 \mathrm{~h}$, and the following hypothesis has been advanced to explain part of these data. Low levels of aversion, such as those provoked by mild or far-away threatening stimuli trigger behavioral mechanisms that mediate more static behavior, such as freezing, while stronger or nearer stimuli provoke more energetic behavior, such as flight or fight. This is supported by the concept that defensive behaviors are hierarchically organized and different behaviors within this class are provoked by aversive stimuli of different intensities (Blanchard \& Blanchard, 1987; Blanchard, Flannelly, \& Blanchard, 1986; Deakin \& Graeff, 1991; Hediger, 1968; Ratner, 1967). In this sense, it would be more adaptive for a prey to hide and freeze when a predator is far away (mild aversive stimulus) rather then engage in flight behavior, which could attract the predator's attention. By the same token, freezing would be useless when the prey is detected and the predator is nearer, flight being a more adaptive reaction.

Furthermore, there is evidence showing that rats switch from active (e.g., turning and horizontal and vertical exploring) to static (freezing) to explosive behaviors (e.g., galloping and jumping) with increasing intensities of aversion (Blanchard \& Blanchard, 1988; Coimbra \& Brandão, 1993; Fanselow, 1991). Thus, the apparent decrease in aversion seen in the present experiment could, in fact, result from switching to a more energetic set of defensive behaviors due to the increase in the aversiveness of the experimental situation by virtue of the addition of social isolation to cart transportation.

Consistent with this hypothesis is the report by Anseloni, Motta, Lima, and Brandão (1995) that rats exposed to an elevated plus-maze with transparent closed walls, in which height is more prominent and probably more aversive, also showed higher exploratory activity than did those tested in a conventional maze with wooden walls. In addition, Quartermain, Stone, and Charbeneau (1994) reported that Swiss Webster mice submitted to four different kinds of stress for $1 \mathrm{~h}$ showed a significantly faster mean latency to emerge from a small dark safe place into a novel, potentially dangerous, large, brightly lit open field. Their locomotor activity and rearing, however, decreased as compared with that of nonstressed control animals. This stresses the notion that some conditions may produce specific changes in particular components of the defense pattern, as suggested by Yudko, Bjornson, and Blanchard (1993).

One conclusion that can be drawn from the present data is that exploratory behaviors directed to the open arms are not the only ones affected by changes in aversiveness; closed-arm measures are also deeply affected. Another has methodological importance, since carts are 
widely used in laboratories everywhere to carry animals from vivaria to testing rooms. The present experiment shows that such a way of transporting may affect the behavioral performance of rats in tests investigating anxiety, such as those using the elevated plus-maze. To further support these suggestions, it would be interesting to perform other experimental manipulations, such as ones using different types of anxiolytic drugs, to assess the kind of aversiveness that is provoked by cart transportation.

\section{REFERENCES}

Anseloni, V. Z., Motta, V., Lima, G., \& Brandão, M. L. (1995). Behavioral and pharmacological validation of the elevated plus maze constructed with transparent walls. Brazilian Journal of Medical \& Biological Research, 28, 597-601.

Blanchard, R. J., \& Blanchard, D. C. (1987). An ethoexperimental approach to the study of fear. Psychological Record, 37, 305-316.

Blanchard, R. J., \& Blanchard, D. C. (1988). Ethoexperimental approaches to the biology of emotion. Annual Review of Psychology, 39, 43-68.

Blanchard, R. J., Flannelly, K. J., \& Blanchard, D. C. (1986). Defensive behaviors of laboratory and wild Rattus norvegicus. Journal of Comparative Psychology, 100, 101-107.

Bruning, J. L., \& KinTz, B. L. (1977). Computational handbook of statistics. Glenview, IL: Scott, Foresman.

Colmbra, N. C., \& Brandão, M. L. (1993). GABAergic nigrocollicular pathways modulate the defensive behavior elicited by midbrain tectum stimulation. Behavioral \& Brain Research, 59, 131-139.

Critchley, M. A. E., \& Handley, S. L. (1987). Effects in the X-maze anxiety model of agents acting at $5-\mathrm{HT}_{1}$ and $5-\mathrm{HT}_{2}$ receptors. Psychopharmacology, 93, 502-506.

Cruz, A. P. M., Frei, F., \& Graeff, F. G. (1994). Ethopharmacological analysis of rat behavior on the elevated plus-maze. Pharmacology, Biochemistry \& Behavior, 49, 171-176.

DeAKIN, J. F. W., \& GraefF, F. G. (1991). 5-HT and mechanisms of defence. Psychopharmacology, 5, 305-315.

FANSELOW, M. S. (1991). The midbrain periaqueductal gray as a coordinator of action in responses to fear and anxiety. In A. De Paulis \& R. Bandler (Eds.), The midbrain periaqueductal gray matter: Functional, anatomical and immunohistochemical organization (pp. 151-173). New York: Plenum.

File, S. E., \& ZANGRossi, H., JR. (1993). "One-trial tolerance" to the anxiolytic actions of benzodiazepines in the elevated plus-maze, or the development of a phobic state? Psychopharmacology, 110, 240244.

Griebel, G., Moreau, J. L., Jenk, F, Martin, J. R., \& Misslin, R. (1993). Some critical determinants of the behaviour of rats in the elevated plus-maze. Behavioural Processes, 29, 37-48.

HANDLEY, S. L., \& Mithani, S. (1984). Effects of alpha-adrenoceptor agonists and antagonists in a maze-exploration model of 'fear'motivated behaviour. Naunyn-Schmiedeberg's Archives of Pharmacology, 327, 1-5.

HEDIGER, H. (1968). The psychology of animals in zoos and circuses. New York: Dover.

Jankowska, E., Pucilowski, O., \& Kostowski, W. (1991). Chronic oral treatment with diltiazem or verapamil decreases isolationinduced activity impairment in elevated plus-maze. Behavioral Brain Research, 43, 155-158.

Johnston, A. L., \& File, S. E. (1991). Sex differences in animal tests of anxiety. Physiology \& Behavior, 49, 245-250.

Maisonnette, S., Morato, S., \& Brandão, M. L. (1993). Role of resocialization and of $5-\mathrm{HT}_{1 \mathrm{~A}}$ receptor activation on the anxiogenic effects induced by isolation in the elevated plus-maze test. Physiology \& Behavior, 54, 753-758.

Montgomery, K. C. (1955). The relation between fear induced by novel stimulation and exploratory behavior. Journal of Comparative \& Physiological Psychology, 48, 254-260.

Morato, S., \& Brandão, M. L. (in press). Paradoxical increase of exploratory behavior in the elevated plus maze by rats exposed to two kinds of aversive stimuli. Brazilian Journal of Medical \& Biological Research.

Morato, S., \& Castrechini, P. (1989). Effects of floor surface and environmental illumination on exploratory activity in the elevated plus-maze. Brazilian Journal of Medical \& Biological Research, 22, 707-710.

Motta, V., Maisonnette, S., Morato, S., Castrechini, P., \& BranDÃO, M. L. (1992). Effects of blockade of 5-HT 2 receptors and activation of $5-\mathrm{HT}_{1 \mathrm{~A}}$ receptors on the exploratory activity of rats in the elevated plus-maze. Psychopharmacology, 107, 135-139.

Pellow, S., Chopin, P., File, S. E., \& Briley, M. (1985). Validation of open:closed arm entries in an elevated plus-maze as a measure of anxiety in the rat. Journal of Neuroscience Methods, 14, 149-167.

Pellow, S., \& File, S. E. (1986). Anxiolytic and anxiogenic drug effects on exploratory activity in an elevated plus-maze: A novel test of anxiety in the rat. Pharmacology. Biochemistry \& Behavior, 24, 525-529.

Quartermain, D., Stone, E. A., \& Charbeneau, G. (1994). Acute stress disrupts defensive behavior in mice. Society for Neuroscience Abstracts, 20, 1445.

RATNER, S. C. (1967). Comparative aspects of hypnosis. In J. E. Gordon (Ed.), Handbook of clinical and experimental hypnosis (pp. 550587). New York: Macmillan.

Treit, D., Menard, J., \& Royan, C. (1993). Anxiogenic stimuli in the elevated plus-maze. Pharmacology, Biochemistry \& Behavior, 44, 463-469.

YudKo, E. B., BJornson, C., \& Blanchard, R. J. (1993). Behavioral differences of dominant and subordinate rats in a visible burrow system. Society for Neuroscience Abstracts, 19, 1620.

(Manuscript received October 17, 1995; revision accepted for publication February 28, 1996.) 